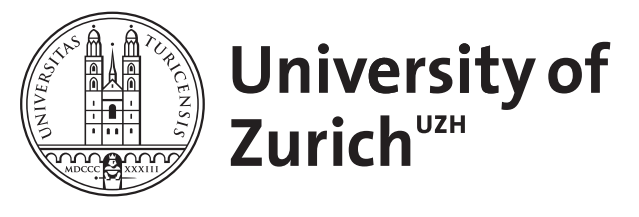

\title{
How Cells Respond to DNA Breaks in Mitosis
}

Blackford, Andrew N ; Stucki, Manuel

\begin{abstract}
DNA double-strand breaks (DSBs) are highly toxic lesions that can lead to chromosomal instability if they are not repaired correctly. DSBs are especially dangerous in mitosis when cells go through the complex process of equal chromosome segregation into daughter cells. When cells encounter DSBs in interphase, they are able to arrest the cell cycle until the breaks are repaired before entering mitosis. However, when DSBs occur during mitosis, cells no longer arrest but prioritize completion of cell division over repair of DNA damage. This review focuses on recent progress in our understanding of the mechanisms that allow mitotic cells to postpone DSB repair without accumulating massive chromosomal instability. Additionally, we review possible physiological consequences of failed DSB responses in mitosis.
\end{abstract}

DOI: https://doi.org/10.1016/j.tibs.2019.12.010

Posted at the Zurich Open Repository and Archive, University of Zurich ZORA URL: https://doi.org/10.5167/uzh-196516

Journal Article

Accepted Version

Originally published at:

Blackford, Andrew N; Stucki, Manuel (2020). How Cells Respond to DNA Breaks in Mitosis. Trends in Biochemical Sciences, 45(4):321-331.

DOI: https://doi.org/10.1016/j.tibs.2019.12.010 


\title{
How cells respond to DNA breaks in mitosis
}

\author{
Andrew N. Blackford ${ }^{1,2}$ and Manuel Stucki ${ }^{3}$
}

${ }^{1}$ Department of Oncology, Medical Research Council Weatherall Institute of Molecular Medicine, University of Oxford, John Radcliffe Hospital, Oxford OX3 9DS, UK

${ }^{2}$ Cancer Research UK/Medical Research Council Oxford Institute for Radiation Oncology, University of Oxford, Oxford OX3 7DQ, UK

${ }^{3}$ Department of Gynecology, University of Zurich, Wagistrasse 14, CH-8952 Schlieren, Switzerland

Keywords: mitosis, DNA double-strand breaks, cell cycle checkpoints, chromosomal instability, micronuclei, chromothripsis, 


\begin{abstract}
DNA double-strand breaks (DSBs) are highly toxic lesions that can lead to chromosomal instability if they are not repaired correctly. DSBs are especially dangerous in mitosis when cells go through the complex process of equal chromosome segregation into daughter cells. When cells encounter DSBs in interphase they are able to arrest the cell cycle before entering mitosis until the breaks are repaired. However, when DSBs occur during mitosis, cells no longer arrest but prioritize completion of cell division over repair of DNA damage. This review focusses on recent progress in our understanding of the mechanisms that allow mitotic cells to postpone DSB repair without accumulating massive chromosomal instability. Additionally, we review possible physiological consequences of failed DSB responses in mitosis.
\end{abstract}




\section{Different responses to DNA breaks in interphase and mitosis}

Cells must maintain genome stability during cycles of cell division in order to pass on their hereditary material intact to the next generation [1]. Although DNA is a relatively stable molecule, it is nonetheless vulnerable to endogenous and exogenous sources of damage. Cells have therefore evolved a complex network of biochemical pathways to counteract these threats, collectively called the DNA damage response (DDR). Activation of the DDR involves recognition and repair of DNA lesions, modulation of chromatin structure and transcription, and cell cycle checkpoint induction [2]. Cell fate upon genotoxic stress in multicellular organisms is also ultimately controlled by the DDR, which determines whether cells re-enter the cell cycle, or permanently exit it (senescence) or undergo programmed cell death (apoptosis).

The vast majority of human cell-based DDR studies have been performed on asynchronous cell populations, with any influence of cell cycle usually studied in the context of comparing how cells respond to DNA damage in specific stages of interphase (G1, S, G2). Far less is understood about how cells cope with genotoxic stress during mitosis, when chromatin becomes dramatically condensed, the nuclear envelope separating nucleus and cytoplasm has broken down, and cells are coordinating the highly complex process of equal chromosome segregation into daughter cells [3]. This lack of understanding is in part due to the difficulties associated with studying a highly dynamic process that can be completed in less than an hour [4], with only a small percentage of cells in an asynchronous population at any one time attempting it. In this review, we describe recent advances in our understanding of how cells respond to DNA breaks in mitosis, highlight the differences in the cellular responses to DNA breaks in interphase and mitosis and identify key questions that remain to be addressed.

\section{DNA damage checkpoint signalling in interphase and mitosis}

Many types of DNA damage can be repaired rapidly and relatively easily without the need for cells to activate cell cycle checkpoints or sustain other global signalling responses. However, some lesions such as DNA double-strand breaks (DSBs) are highly toxic and can be particularly challenging for cells to repair accurately without causing genome instability [1], especially if left unrepaired when cells attempt chromosome segregation during mitosis. The DDR is initiated by three structurally related protein kinases: ATM, ATR and DNA-PKcs [5]. ATM and DNA-PKcs are primarily activated by DSBs, whereas ATR can be activated by lesions that are processed to produce single-stranded DNA (ssDNA). The primary role of DNA-PKcs is probably limited to DSB repair in the vicinity of the lesion, whereas ATM and 
ATR activate both local and global cellular responses to DNA damage, in part by phosphorylating and activating other kinases such as CHK1 and CHK2, respectively. However, it is important to note that ATM, ATR and DNA-PKcs have a range of overlapping substrates and can substitute for each other to some extent.

All three DDR kinases are recruited and activated by a discrete set of co-factors. ATM is recruited and activated by the MRE11-RAD50-NBS1 (MRN) complex, which can recognise and bridge broken double-stranded DNA (dsDNA) ends [6,7]; DNA-PKcs is recruited and activated at dsDNA ends by $\mathrm{Ku}$, a heterodimeric complex consisting of two subunits, $\mathrm{Ku} 70$ and Ku80 [8]; and ATR has a stable binding partner, ATRIP [9], which binds to the heterotrimeric RPA complex when it is bound to ssDNA [10]. ATM and DNA-PKcs are activated by binding to their co-factors at DSB ends, but ATR requires additional stimulation by either TOPBP1 or ETAA1, which both contain an ATR-activation domain [11-13]. ETAA1 is recruited directly to RPA-coated ssDNA where it can activate ATR kinase activity, whereas the factors and mechanisms controlling TOPBP1 recruitment are not yet clear but may involve multiple, possibly redundant mechanisms [14-16].

In response to DNA damage, ATM and ATR activate cell cycle checkpoints to arrest cells at the G1/S or G2/M boundaries [17]. In contrast, S phase checkpoint signalling limits DNA replication origin firing, stabilises stalled replication forks and increases nucleotide supply to slow S phase progression and maintain the fidelity of DNA replication rather than causing cells to arrest [18]. While there are differences in how these checkpoints are activated, they all share essentially the same output, i.e. inhibition of the cyclin-dependent kinase (CDK) activity that drives cell cycle progression. This is achieved rapidly by ATM/ATR-dependent activation of checkpoint kinases that inactivate the CDC25 family of phosphatases, which counteract inhibitory phosphorylation of CDKs by the WEE1 kinase. A slower, transcriptiondependent inhibition of CDKs by 21 is also activated by ATM- and ATR-dependent phosphorylation of $\mathrm{p} 53$, which is particularly important for the G1/S checkpoint.

What about DNA damage checkpoint signalling in mitosis? Once cells have moved beyond prophase, there is no cell cycle checkpoint until daughter cells re-enter G1 in the next cell cycle [19]. While high levels of DNA damage can delay the metaphase-to-anaphase transition, this is due to defects in microtubule attachment to kinetochores and an active spindle assembly checkpoint, rather than direct signalling from DNA lesions [20].

It is therefore clear that cells at some point become committed to mitosis and prioritize completion of cell division over repair of DNA damage. There are several mechanisms that prevent inhibition of mitosis driving CDK1 by DNA damage signalling. Firstly, little or no 
transcription occurs during mitosis except possibly at specific genes and regions such as centromeres [21-23], so mechanisms such as p21 induction and subsequent inhibition of CDKs are disabled. Another major mechanism involves ubiquitylation and proteasomal degradation of key cell-cycle and DNA damage checkpoint mediators. WEE1 is phosphorylated by CDK1 and PLK1 at the onset of mitosis, which creates phospho-degrons that are recognized by the SCF- $\beta$ TrCP ubiquitin ligase, resulting in WEE1 degradation [24]. Claspin, a protein required for CHK1 activation [25], is also phosphorylated by PLK1 and degraded by SCF- $\beta$ TrCP [2628]. Finally, CHK2 is phosphorylated and inhibited by PLK1 upon mitotic entry, which prevents CHK2 activation rather than promoting its degradation [29]. ATM-dependent phosphorylation of CHK2 is also reduced during mitosis, which may further limit CHK2 kinase activity $[29,30]$. This may be required because aberrant ATM-CHK2 signalling during mitosis can result in stabilisation of kinetochore-microtubule attachments to chromosomes, thereby increasing the frequency of lagging chromosomes during anaphase [31]. Taken together, CDK1-Cyclin B activity is thus maximised at the end of prophase through multiple mechanisms and can no longer be inhibited by ATM or ATR signalling until mitosis is complete.

Interestingly, there is a small time window between antephase (defined as the time point after centrosome separation occurs but before chromosomes condense) and late prophase, where even a small amount of DNA damage triggers cells to decondense their chromosomes and to return to antephase [19], where they permanently exit the cell cycle [32]. It appears that the DNA damage threshold for re-entering the cell cycle is lower than for the G2/M checkpoint, with more cells becoming senescent when irradiated in antephase compared to G2 at similar doses of genotoxic stress [32]. This response is ATM-dependent [33], and appears to be mechanistically distinct from the previously described "antephase checkpoint" [34], which is reversible and activated by spindle poisons, and depends on CHFR and p38 MAP kinases but not ATM/ATR signaling [33-36].

\section{Chromatin responses to DNA breaks in interphase and mitosis}

Even though no cell cycle checkpoints appear to exist in mitosis after cells have entered prophase, the ATM and DNA-PKcs kinases are still activated by DSBs in mitosis and their activation appears to be essential for cell survival in response to mitotic DNA breaks [30]. Besides activation of cell cycle checkpoints, cells also rely on ATM activity to regulate DSB repair pathway choice. Two main pathways repair DSBs: non-homologous end-joining (NHEJ) 
and homologous recombination (HR). NHEJ re-ligates DNA ends with limited or no DNA end processing and operates throughout interphase, but appears to be inhibited in mitosis $[37,38]$ (see also below). HR pathways rely on homology and templated DNA synthesis to regenerate the sequence surrounding the break site. In order to avoid loss of heterozygosity, HR is restricted to $\mathrm{S}$ and $\mathrm{G} 2$ phases of the cell cycle where the sister-chromatid is used as template for repair synthesis [38]. DNA-end resection is a prerequisite for HR, and the extent to which it is allowed to proceed is one of the factors that determines DSB repair pathway choice. Not surprisingly, DNA-end resection is a tightly regulated process that is restricted to S and G2 phases of the cell cycle due to CDK-dependent phosphorylation of the pro-resection factor CtIP $[39,40]$. Key regulatory mechanisms that limit DNA-end resection in G1 and mitosis also operate at the level of chromatin modifications in regions flanking the DSB site [41].

Chromatin is extensively modified by post-translational modifications in large regions spanning several hundred kilobases of DNA flanking both sides of a DSB. These regions form cytologically discernible subnuclear structures that are referred to as nuclear foci, DNA damage foci, or ionizing radiation (IR)-induced foci (IRIF; [42]). The key regulators of IRIF formation are histone proteins that form the core of the nucleosome, the organizational unit of eukaryotic genomes. In response to DSBs, the histone $\mathrm{H} 2 \mathrm{~A}$ variant $\mathrm{H} 2 \mathrm{AX}$, a component of the nucleosome core structure that comprises $10-15 \%$ of total cellular $\mathrm{H} 2 \mathrm{~A}$ in higher organisms, is rapidly phosphorylated by ATM and DNA-PKcs on a conserved Ser residue (S139) at its Cterminus. Phosphorylated H2AX (termed $\gamma \mathrm{H} 2 \mathrm{AX}$ ) "spreads" over large chromatin domains throughout the cell cycle but is strictly confined to the damaged chromosome and does not involve neighboring chromosomes that are not affected by DNA damage [43]. The phosphorylated H2AX C-terminus serves as a chromatin mark that flags regions in the genome that contain DNA breaks. MDC1, a large protein that belongs to the mediator/adaptor group of DDR factors, specifically binds to the phosphorylated H2AX C-terminus and appears to be the predominant $\gamma \mathrm{H} 2 \mathrm{AX}$ recognition factor in mammalian cells [44]. MDC1 mediates the recruitment and activation of the ubiquitin ligase RNF8 via direct interaction [45-47]. RNF8 ubiquitylates the linker histone $\mathrm{H} 1$ in chromatin regions flanking DSBs and thus generates an additional histone mark that recruits and activates another ubiquitin ligase, RNF168 [48]. RNF168 mono-ubiquitylates H2A-type histones on Lys-15 [49,50], thus generating a binding site for 53BP1, a mediator protein that limits DNA-end resection to promote the fidelity of DSB repair [51,52]. 53BP1 binds to ubiquitylated H2A-type histones via an unusual ubiquitin recognition motif ensuring its specific binding to damaged chromatin marks [51]. In addition, 53BP1 contains a Tudor domain that binds specifically to histone $\mathrm{H} 4$ when it is di-methylated 
on Lys-20 (H4K20me2) [53], thus making 53BP1 a bivalent histone mark reader [54]. Upon accumulation around DSBs, 53BP1 recruits various effector proteins, including RIF1 [55-58], PTIP [59], and the recently discovered Shieldin-CST complex that limits end resection to promote the fidelity of DSB repair by NHEJ and HR over mutagenic pathways such as singlestrand annealing [52,60-70] (Figure 1A).

In mitosis, $\mathrm{H} 2 \mathrm{AX}$ is also phosphorylated at sites of DSBs and is readily recognized by MDC1 [30]. However, part of the signaling cascade downstream of MDC1 that regulates DSB repair pathway choice is interrupted in mitotic cells. For example, RNF8, RNF168, 53BP1 and BRCA1 are not recruited during mitosis $[29,30,71,72]$. It was proposed that two roadblocks inhibit recruitment of these proteins: first, a cell cycle-dependent inhibition of the RNF8MDC1 interaction blocks the initiation of chromatin ubiquitylation [73]; and second, the phosphorylation of 53BP1 on two residues that inhibits its interaction with ubiquitylated $\mathrm{H} 2 \mathrm{~A} / \mathrm{H} 2 \mathrm{AX}$ and thus blocks 53BP1 accumulation on damaged chromatin $[73,74]$. As a result, chromatin ubiquitylation is abrogated in early mitosis, but can occur in late anaphase and telophase [75]. 53BP1 recruitment on the other hand is dependent on the removal of the inhibitory phosphorylations by protein phosphatase 4C (PP4C) and does not occur until cells enter G1 [74,75]. The striking truncation of the chromatin response to DSBs in mitosis thus raises the question of how DNA breaks are dealt with in this crucial phase of the cell cycle (Figure 1B).

\section{Are DSBs repaired in mitosis?}

The absence of 53BP1 recruitment to sites of DSBs in mitosis removes the DNA-end resection roadblock and could in theory allow over-resection in mitosis. However, this is not observed in mitotic cells [76,77], possibly due to the fact that chromosomes are too highly condensed for enzymes involved in long-range resection to overcome. In addition to lack of DNA-end resection, key HR factors such as BRCA1 and RAD51 are also not recruited to mitotic DSB sites $[30,78]$. The bulk of evidence therefore suggests that DSB repair by HR is inactive in mitosis. A lack of RPA-coated ssDNA in mitosis might also explain why ATR is not activated by DSBs during mitosis, although ATR may be activated during mitosis by other stimuli [79].

The situation is less clear when it comes to NHEJ: while early studies suggested that DSB repair is universally inhibited during mitosis [19,80], and that therefore DSBs are also not repaired by NHEJ until the following G1 phase, the absence of NHEJ during mitosis has never been demonstrated directly. In fact, there is circumstantial evidence arguing that although chromatin responses are attenuated during mitosis (see above), the DSB repair machinery itself 
may remain active to some extent. For example, $\mathrm{Ku}$ foci still form on mitotic chromatin $[81,82]$, although it is unclear if they are resolved during mitosis or in the following G1. Given that expression of 53BP1 mutants that cannot be phosphorylated by mitotic kinases promote sistertelomere fusions [73], it seems likely that at least some NHEJ activity is present in mitotic cells. On the other hand, there is evidence that XRCC4 is phosphorylated by mitotic kinases and that this attenuates its localization at DSB sites [83]. Thus, it is still unclear to what extent the core NHEJ machinery is inactivated during mitosis.

Regardless, in the absence of efficient DSB repair, broken chromosomes represent a major threat for mitotic cells since broken chromatid fragments lacking a centromere (so called acentric fragments) would be unable to segregate properly without the existence of compensatory mechanisms. A simple such mechanism would be tethering of broken chromosome ends until they can be repaired in the following G1 phase. Evidence for the existence of a tethering mechanism has been discovered in Drosophila melanogaster, where acentric chromatid fragments segregate efficiently to opposite poles because of a DNA tether that is covered with the mitotic kinases Polo (PLK1 in vertebrates), Aurora B and BubR1 [84] (Figure 2A). It appears that one consequence of BubR1 localization on these DNA tethers is the local inhibition of $\mathrm{APC} / \mathrm{C}$ complex, which may be required for proper transmission of broken chromosomes in Drosophila [85]. It is currently not clear if DSBs are similarly stabilized during mitosis in mammalian cells, but inhibition of the $\mathrm{APC} / \mathrm{C}$ is the outcome of the spindle checkpoint, and it is known that in human cells this checkpoint is not activated by DSBs. However, recent evidence suggests that tethering of acentric chromatid fragments during mitosis may at least exist in mammalian cells [15]. As described above, the chromatin response to DSBs is only partially disrupted in mitosis, such that the upstream events such as $\mathrm{H} 2 \mathrm{AX}$ phosphorylation and MDC1 recruitment still occur. It appears that a central purpose of the chromatin response to DSBs during mitosis is the recruitment of the mediator protein TOPBP1 [15]. TOPBP1 is a versatile adaptor protein with multiple roles in DNA replication, DNA repair and transcription [16]. In mitosis, TOPBP1 is recruited to sites of DSBs via direct phosphorylation-dependent interaction with MDC1. Disruption of this interaction results in chromosomal instability that likely originates from errors in mitosis [15]. Interestingly, TOPBP1 accumulates at sites of DSBs in mitosis in filamentous assemblies that frequently bridge two MDC1 IRIF either within (intra) or occasionally between (inter) chromosomes, indicating that they may represent structures that are tethering broken chromosomes [15] (Figure 2B). Whether or not these tethers are related to the BubR1-coated DNA tethers observed in Drosophila is currently not clear, but it is interesting to note that Drosophila strains 
lacking the MDC1 orthologue MU2 show high-frequencies of terminal deficiencies (i.e. chromosomes that have lost the tip regions) [86], which would be an expected outcome of defective tethering of acentric fragments in mitosis and are also observed in human cells lacking MDC1-TOPBP1 interaction [15].

\section{Consequences of defective responses to DNA breaks during mitosis}

If a DSB that occurs in mitosis fails to be repaired or tethered, the affected chromosome breaks up into two pieces: an acentric chromosome fragment and a centric chromosome fragment, each containing a telomere on one end, and free DNA end on the other (Figure 3). Since the acentric fragment is not able to interact with the mitotic spindle, it lingers near the equatorial plate during anaphase. These acentric fragments usually randomly segregate into the cytoplasm of one of the daughter cells and are converted into micronuclei (see below).

The centric fragment on the other hand is able to attach to the spindle and can thus be properly segregated into one of the daughter cells, but its free DNA end can potentially fuse to another centric fragment of another chromosome or to an intact chromosome with a deprotected telomere in the subsequent G1, leading to the formation of a dicentric chromosome $[87,88]$. These dicentric chromosomes are source of so-called breakage-fusion-breakage (BFB) cycles, first described by Barbara McClintock in 1941 [89]. BFB cycles are a major source of chromosomal instability in cancer.

Micronuclei on the other hand are associated with heavily deregulated DNA metabolism and can thus give rise to a unique form of chromosomal instability termed chromothripsis [90], where large numbers of clustered chromosomal rearrangements occur in a confined genomic region typically only on one chromosome. Chromothripsis can occur when a micronucleus is reincorporated in the main nucleus in one of the subsequent mitosis [91]. Thus, failed responses to DNA breaks in mitosis can critically contribute to the formation of the kind of chromosomal instability frequently observed in cancers (Figure 3).

It is well established that genotoxic stress leads to inflammatory responses. For example, exposure of cells to DSB-inducing agents leads to the expression of type I interferons, which activates p53 and induces senescence [92]. It was originally suggested that DNA fragments leaking from the site of DNA damage in the nucleus into the cytosol are recognized by cyclic GMP-AMP synthase (cGAS), an important sensor of cytosolic DNA that activates innate immunity by engaging the stimulator of interferon genes (STING) cascade $[93,94]$. However, two recent studies identified micronuclei as the main source of immune stimulatory cytosolic DNA $[95,96]$. Even though micronuclei are structurally comparable to primary nuclei, their 
nuclear envelope (NE) is unstable, resulting in frequent NE breakdown [97], which leads to the exposure of fragmented DNA from within the micronuclei to cGAS and activates an inflammatory response (Figure 3). Thus, a failed response to DNA breaks in mitosis may have physiological consequences beyond accumulation of chromosomal instability and may contribute to shape the immune microenvironment of tumors.

\section{Concluding remarks}

The key features of the specialized response to DNA breaks in mitosis can be summarized as follows. Upon entering prophase, cells do not arrest the cell cycle in the presence of DSBs and instead prioritize the completion of cell division over the repair of DNA breaks. The chromatin response to DSBs is truncated in mitosis. Even though $\mathrm{H} 2 \mathrm{AX}$ is efficiently phosphorylated in mitosis and is readily recognized by MDC1, recruitment of key downstream factors implicated in the regulation of DSB repair pathway choice such as 53BP1 and BRCA1 is blocked. In addition, DNA-end resection, a pre-requisite for DSB repair by HR, is limited. Instead, cells utilize a pathway to tether broken chromosomes that involves recruitment of TOPBP1 by direct interaction with MDC1, which is critical for the suppression of chromosomal instability during mitosis. A failed DSB response in mitosis results in micronuclei formation and BFB cycles. Micronuclei are associated with deregulated DNA metabolism and frequent NE breakdown, giving rise to chromothripsis and the activation of innate immunity via the cGAS-STING pathway.

Despite these recent insights, one of the key outstanding questions that remains unanswered (see Outstanding questions box) is if DSBs that occur during mitosis are repaired or tethered until NHEJ is reactivated in the following G1. Indirect evidence exists for both options and it is formally possible (perhaps even likely) that DSBs in mitosis can be either tethered or repaired, depending on the context of when and where they occur and whether they have compatible (clean) ends for repair. For example, it is possible that DSBs that occur early in mitosis (prophase or metaphase) are mostly repaired, while DSBs that occur in later stages (anaphase or telophase) are preferentially tethered for repair in G1. Regardless, it is clear that TOPBP1 is a key factor in the regulation of the mitotic DSB response. Therefore, it will be essential to define the molecular mechanisms by which it suppresses chromosomal instability in mitosis. This will include the detailed characterization of how it is recruited to sites of DSBs in mitosis as well as the identification of downstream factors that are regulated by the MDC1TOPBP1 complex. A deeper mechanistic understanding of how cells deal with DNA breaks in mitosis may breed new ideas how to exploit these phenomena for anti-cancer therapies. 


\section{Figure legends}

Figure 1: Rewiring of the phosphorylation-dependent protein interaction network at damaged chromatin regions in mitosis.

(A) Phosphorylation-dependent protein interaction network in interphase, recruiting the 53BP1-Shieldin complex and the BRCA1-A complex to damaged chromatin regions via activation of the $\gamma \mathrm{H} 2 \mathrm{AX}-\mathrm{MDC} 1-\mathrm{RNF} 8-\mathrm{RNF} 168$ chromatin ubiquitylation cascade.

(B) Early in mitosis, CDK1-dependent phosphorylation of RNF8 inhibits its interaction with MDC1 and leads to the abrogation of the chromatin ubiquitylation cascade. In addition, mitotic kinases inactivate the ubiquitin-dependent chromatin interaction domain of 53BP1. TOPBP1, which is predominantly recruited by 53BP1 in G1, is directly recruited by $\mathrm{MDC} 1$ in mitosis.

Figure 2: Potential DSB tethering mechanisms in Drosophila and mammalian cells.

(A) DSBs induced by the I-Cre1 endonuclease in the rDNA repeats of chromosome $\mathrm{X}$ in Drosophila cells produce acentric fragments that are efficiently segregating poleward due to DNA tethers that connect them to the centric fragments. These DNA tethers are decorated with the mitotic kinases BubR1, Polo and Aurora-B, as well as INCENP. Downregulation of BubR1 and Polo function results in acentric segregation defects.

(B) IR-induced breaks in prometaphase chromosomes in human cells lead to $\gamma \mathrm{H} 2 \mathrm{AX}$ formation and recruitment of MDC1. Through direct phosphorylation-dependent interaction, MDC1 mediates the accumulation of filamentous TOPBP1 assemblies at sites of mitotic DSBs. These TOPBP1 filaments frequently bridge two MDC1 IRIF and may thus represent tethering structures. Disruption of MDC1-TOPBP1 complex formation leads to defective TOPBP1 recruitment in mitosis and chromosomal instability.

\section{Figure 3: Physiological consequences of a failed response to DNA breaks in mitosis.}

If DSBs are not tethered or repaired in mitosis, acentric fragments are not segregated and lead to micronuclei formation. DNA metabolism is heavily disturbed in micronuclei, which results in chromatin fragmentation. If a micronucleus is reincorporated in the main nucleus, it may induce chromothripsis. In addition, the 
nuclear envelopes of micronuclei are unstable, which allows exposure of fragmented chromatin to cGAS, a cytosolic sensor of DNA. This leads to an inflammatory response via activation of STING-dependent type I interferon production.

Centric fragments from unrepaired DSBs during mitosis are properly segregated but may result in terminal deficiencies or formation of dicentric chromosome, which in the subsequent cell cycles, lead to anaphase chromosome bridges and BFB cycles. 


\section{Glossary box}

Interphase: the segment of the cell cycle that lies between two cell divisions. In interphase the chromatin is replicated and the cell prepares for cell division. It is sub-divided in synthesis phase (S phase) and two gap phases (G1 and G2).

Senescence: irreversible cell cycle arrest characterized by alterations in gene expression patterns and genome organization.

Nuclear envelope: two lipid bilayer membranes surrounding the cell nucleus and encasing the genetic material. Nuclear pores in the nuclear envelope allow exchange of materials between the cytosol and the nucleus.

Cell cycle checkpoints: control mechanisms in eukaryotic cells that block or slow cell cycle progression to ensure proper cell division in response to stress. DNA damage checkpoints are the G1/S checkpoint, the intra-S phase checkpoint and the G2/M checkpoint.

Chromosome segregation: a mitotic process in which the two sister chromatids separate from each other and migrate to opposite poles of the nucleus.

Centromere: a specialized DNA sequence on chromosomes where the sister chromatids are paired together. During mitosis, spindle fibres attach to the centromere via the kinetochore.

Kinetochore: a disc-shaped protein structure associated with centromeres where the spindle fibres attach during cell division to pull sister chromatids apart.

Spindle assembly checkpoint: a cell cycle checkpoint that prevents separation of the sister chromatids until each chromosome is properly attached to the spindle apparatus.

DNA-end resection: nucleolytic processing of DNA ends at sites of DSBs that generates 3' single-stranded DNA overhangs necessary to induce the process of homologous recombination.

Ionizing radiation: types of radiation (including X-rays or gamma rays) that carry sufficient energy to detach electrons from molecules, thus ionizing them.

Mediator/adaptor: a protein that mediates the interaction between two proteins that are unable to interact directly with each other.

Sister telomeres: telomeres at the tips of the paired sister chromatids. In early phases of mitosis, sister telomeres are located in close proximity to each other.

Anaphase-promoting complex/cyclosome (APC/C): a multi-subunit E3 ubiquitin ligase complex that targets various cell cycle proteins for degradation by the proteasome and thus mainly regulates metaphase to anaphase transition.

Mitotic spindle: a cytoskeletal structure mostly composed of microtubules that forms during mitosis to separate sister chromatids. 
Dicentric chromosome: abnormal chromosome that contains two centromeres. They can form by fusion of two chromosome segments that each contain a centromere or by intrachromosomal recombination and give rise to anaphase chromosome bridges and breakagefusion-breakage cycles.

Type I interferons: a group of signalling proteins of the innate immune system mainly important for fighting viral infections. Type I interferons bind to the specific cell surface receptor IFN- $\alpha / \beta$. 


\section{References}

1 Jackson, S.P. and Bartek, J. (2009) The DNA-damage response in human biology and disease. Nature 461, 1071-1078

2 Ciccia, A. and Elledge, S.J. (2010) The DNA damage response: making it safe to play with knives. Molecular Cell 40, 179-204

3 Wieser, S. and Pines, J. (2015) The biochemistry of mitosis. Cold Spring Harb Perspect Biol 7, a015776

4 Araujo, A.R. et al. (2016) Positive Feedback Keeps Duration of Mitosis Temporally Insulated from Upstream Cell-Cycle Events. Molecular Cell 64, 362-375

5 Blackford, A.N. and Jackson, S.P. (2017) ATM, ATR, and DNA-PK: The Trinity at the Heart of the DNA Damage Response. Molecular Cell 66, 801-817

6 Lee, J.-H. and Paull, T.T. (2004) Direct activation of the ATM protein kinase by the Mre11/Rad50/Nbs1 complex. Science 304, 93-96

7 Lee, J.-H. and Paull, T.T. (2005) ATM activation by DNA double-strand breaks through the Mre11-Rad50-Nbs1 complex. Science 308, 551-554

8 Gottlieb, T.M. and Jackson, S.P. (1993) The DNA-dependent protein kinase: requirement for DNA ends and association with Ku antigen. Cell 72, 131-142

9 Cortez, D. et al. (2001) ATR and ATRIP: partners in checkpoint signaling. Science 294, 1713-1716

10 Zou, L. and Elledge, S.J. (2003) Sensing DNA damage through ATRIP recognition of RPA-ssDNA complexes. Science 300, 1542-1548

11 Bass, T.E. et al. (2016) ETAA1 acts at stalled replication forks to maintain genome integrity. Nat Cell Biol 18, 1185-1195

12 Haahr, P. et al. (2016) Activation of the ATR kinase by the RPA-binding protein ETAA1. Nat Cell Biol 18, 1196-1207

13 Kumagai, A. et al. (2006) TopBP1 activates the ATR-ATRIP complex. Cell 124, $943-$ 955

14 Blackford, A.N. et al. (2015) TopBP1 Interacts with BLM to Maintain Genome Stability but Is Dispensable for Preventing BLM Degradation. Molecular Cell 57, 1133-1141

15 Leimbacher, P.-A. et al. (2019) MDC1 Interacts with TOPBP1 to Maintain Chromosomal Stability during Mitosis. Molecular Cell 74, 571-583.e8

16 Wardlaw, C.P. et al. (2014) TopBP1: A BRCT-scaffold protein functioning in multiple cellular pathways. DNA Repair (Amst) 22, 165-174

17 Kastan, M.B. and Bartek, J. (2004) Cell-cycle checkpoints and cancer. Nature 432, 316323

18 Chao, H.X. et al. (2017) Orchestration of DNA Damage Checkpoint Dynamics across the Human Cell Cycle. Cell Syst 5, 445-459.e5

19 Rieder, C.L. and Cole, R.W. (1998) Entry into mitosis in vertebrate somatic cells is guarded by a chromosome damage checkpoint that reverses the cell cycle when triggered during early but not late prophase. The Journal of Cell Biology 142, 1013 1022

20 Mikhailov, A. et al. (2002) DNA damage during mitosis in human cells delays the metaphase/anaphase transition via the spindle-assembly checkpoint. Curr Biol 12, 17971806

21 Gottesfeld, J.M. and Forbes, D.J. (1997) Mitotic repression of the transcriptional machinery. Trends Biochem Sci 22, 197-202 
22 Chan, F.L. et al. (2012) Active transcription and essential role of RNA polymerase II at the centromere during mitosis. Proceedings of the National Academy of Sciences 109, 1979-1984

23 Palozola, K.C. et al. (2017) Mitotic transcription and waves of gene reactivation during mitotic exit. Science 358, 119-122

24 Watanabe, N. et al. (2004) M-phase kinases induce phospho-dependent ubiquitination of somatic Wee1 by SCFbeta-TrCP. Proc Natl Acad Sci USA 101, 4419-4424

25 Kumagai, A. and Dunphy, W.G. (2000) Claspin, a novel protein required for the activation of Chk1 during a DNA replication checkpoint response in Xenopus egg extracts. Molecular Cell 6, 839-849

26 Mailand, N. et al. (2006) Destruction of Claspin by SCFbetaTrCP restrains Chk1 activation and facilitates recovery from genotoxic stress. Molecular Cell 23, 307-318

27 Peschiaroli, A. et al. (2006) SCFbetaTrCP-mediated degradation of Claspin regulates recovery from the DNA replication checkpoint response. Molecular Cell 23, 319-329

28 Mamely, I. et al. (2006) Polo-like kinase-1 controls proteasome-dependent degradation of Claspin during checkpoint recovery. Curr Biol 16, 1950-1955

29 van Vugt, M.A.T.M. et al. (2010) A mitotic phosphorylation feedback network connects Cdk1, Plk1, 53BP1, and Chk2 to inactivate the G(2)/M DNA damage checkpoint. PLoS Biol 8, e1000287

30 Giunta, S. et al. (2010) DNA damage signaling in response to double-strand breaks during mitosis. The Journal of Cell Biology 190, 197-207

31 Bakhoum, S.F. et al. (2014) DNA-damage response during mitosis induces wholechromosome missegregation. Cancer Discov 4, 1281-1289

32 Feringa, F.M. et al. (2016) Hypersensitivity to DNA damage in antephase as a safeguard for genome stability. Nature Communications 7, 12618

33 Mikhailov, A. et al. (2004) Topoisomerase II and histone deacetylase inhibitors delay the G2/M transition by triggering the p38 MAPK checkpoint pathway. The Journal of Cell Biology 166, 517-526

34 Matsusaka, T. and Pines, J. (2004) Chfr acts with the p38 stress kinases to block entry to mitosis in mammalian cells. The Journal of Cell Biology 166, 507-516

35 Rieder, C.L. and COLE, R. (2000) Microscopy-induced radiation damage, microtubules, and progression through the terminal stage of G2 (prophase) in vertebrate somatic cells. Cold Spring Harb. Symp. Quant. Biol. 65, 369-376

36 Scolnick, D.M. and Halazonetis, T.D. (2000) Chfr defines a mitotic stress checkpoint that delays entry into metaphase. Nature 406, 430-435

37 Heijink, A.M. et al. (2013) The DNA damage response during mitosis. Mutat Res 750, 45-55

38 Hustedt, N. and Durocher, D. (2016) The control of DNA repair by the cell cycle. Nat Cell Biol 19, 1-9

39 Sartori, A.A. et al. (2007) Human CtIP promotes DNA end resection. Nature 450, 509514

40 Huertas, P. and Jackson, S.P. (2009) Human CtIP mediates cell cycle control of DNA end resection and double strand break repair. J Biol Chem 284, 9558-9565

41 Chapman, J.R. et al. (2012) Playing the end game: DNA double-strand break repair pathway choice. Molecular Cell 47, 497-510

42 Fernandez-Capetillo, O. et al. (2003) Focusing on foci: H2AX and the recruitment of DNA-damage response factors. Cell Cycle 2, 426-427

43 Rogakou, E.P. et al. (1999) Megabase chromatin domains involved in DNA doublestrand breaks in vivo. The Journal of Cell Biology 146, 905-916 
44 Stucki, M. and Jackson, S.P. (2006) gammaH2AX and MDC1: anchoring the DNAdamage-response machinery to broken chromosomes. DNA Repair (Amst) 5, 534-543

45 Huen, M.S.Y. et al. (2007) RNF8 transduces the DNA-damage signal via histone ubiquitylation and checkpoint protein assembly. Cell 131, 901-914

46 Kolas, N.K. et al. (2007) Orchestration of the DNA-damage response by the RNF8 ubiquitin ligase. Science 318, 1637-1640

47 Mailand, N. et al. (2007) RNF8 ubiquitylates histones at DNA double-strand breaks and promotes assembly of repair proteins. Cell 131, 887-900

48 Thorslund, T. et al. (2015) Histone H1 couples initiation and amplification of ubiquitin signalling after DNA damage. Nature 527, 389-393

49 Gatti, M. et al. (2012) A novel ubiquitin mark at the N-terminal tail of histone H2As targeted by RNF168 ubiquitin ligase. Cell Cycle 11, 2538-2544

50 Mattiroli, F. et al. (2012) RNF168 Ubiquitinates K13-15 on H2A/H2AX to Drive DNA Damage Signaling. Cell 150, 1182-1195

51 Fradet-Turcotte, A. et al. (2013) 53BP1 is a reader of the DNA-damage-induced H2A Lys 15 ubiquitin mark. Nature 499, 50-54

52 Ochs, F. et al. (2016) 53BP1 fosters fidelity of homology-directed DNA repair. Nat Struct Mol Biol 23, 714-721

53 Botuyan, M.V. et al. (2006) Structural basis for the methylation state-specific recognition of histone H4-K20 by 53BP1 and Crb2 in DNA repair. Cell 127, 1361-1373

54 Wilson, M.D. et al. (2016) The structural basis of modified nucleosome recognition by 53BP1. Nature 536, 100-103

55 Chapman, J.R. et al. (2013) RIF1 is essential for 53BP1-dependent nonhomologous end joining and suppression of DNA double-strand break resection. Molecular Cell 49, 858871

56 Di Virgilio, M. et al. (2013) Rif1 prevents resection of DNA breaks and promotes immunoglobulin class switching. Science 339, 711-715

57 Zimmermann, M. et al. (2013) 53BP1 regulates DSB repair using Rif1 to control 5' end resection. Science 339, 700-704

58 Escribano-Diaz, C. et al. (2013) A cell cycle-dependent regulatory circuit composed of 53BP1-RIF1 and BRCA1-CtIP controls DNA repair pathway choice. Molecular Cell 49, $872-883$

59 Callen, E. et al. (2013) 53BP1 mediates productive and mutagenic DNA repair through distinct phosphoprotein interactions. Cell 153, 1266-1280

60 Boersma, V. et al. (2015) MAD2L2 controls DNA repair at telomeres and DNA breaks by inhibiting 5 ' end resection. Nature 521, 537-540

$61 \mathrm{Xu}, \mathrm{G}$. et al. (2015) REV7 counteracts DNA double-strand break resection and affects PARP inhibition. Nature 521, 541-544

62 Gupta, R. et al. (2018) DNA Repair Network Analysis Reveals Shieldin as a Key Regulator of NHEJ and PARP Inhibitor Sensitivity. Cell 173, 972-988.e23

63 Tomida, J. et al. (2018) FAM35A associates with REV7 and modulates DNA damage responses of normal and BRCA1-defective cells. EMBO J 37,

64 Ghezraoui, H. et al. (2018) 53BP1 cooperation with the REV7-shieldin complex underpins DNA structure-specific NHEJ. Nature 560, 122-127

65 Noordermeer, S.M. et al. (2018) The shieldin complex mediates 53BP1-dependent DNA repair. Nature 560, 117-121

66 Dev, H. et al. (2018) Shieldin complex promotes DNA end-joining and counters homologous recombination in BRCA1-null cells. Nat Cell Biol 20, 954-965

67 Findlay, S. et al. (2018) SHLD2/FAM35A co-operates with REV7 to coordinate DNA double-strand break repair pathway choice. EMBO J 37, 
68 Gao, S. et al. (2018) An OB-fold complex controls the repair pathways for DNA doublestrand breaks. Nature Communications 9, 3925

69 Barazas, M. et al. (2018) The CST Complex Mediates End Protection at Double-Strand Breaks and Promotes PARP Inhibitor Sensitivity in BRCA1-Deficient Cells. CellReports 23, 2107-2118

70 Mirman, Z. et al. (2018) 53BP1-RIF1-shieldin counteracts DSB resection through CSTand Pola-dependent fill-in. Nature 560, 112-116

71 Nelson, G. et al. (2009) DNA damage foci in mitosis are devoid of 53BP1. Cell Cycle 8, 3379-3383

72 Nakamura, A.J. et al. (2010) The complexity of phosphorylated H2AX foci formation and DNA repair assembly at DNA double-strand breaks. Cell Cycle 9, 389-397

73 Orthwein, A. et al. (2014) Mitosis inhibits DNA double-strand break repair to guard against telomere fusions. Science 344, 189-193

74 Lee, D.-H. et al. (2014) Dephosphorylation enables the recruitment of 53BP1 to doublestrand DNA breaks. Molecular Cell 54, 512-525

75 Giunta, S. and Jackson, S.D. (2011) Give me a break, but not in mitosis: The mitotic DNA damage response marks DNA double strand breaks with early signaling events. Cell Cycle 10,

$76 \mathrm{Xu}, \mathrm{N}$. et al. (2010) Akt/PKB suppresses DNA damage processing and checkpoint activation in late G2. The Journal of Cell Biology 190, 297-305

77 Peterson, S.E. et al. (2011) Cdk1 uncouples CtIP-dependent resection and Rad51 filament formation during M-phase double-strand break repair. The Journal of Cell Biology 194, 705-720

78 Ayoub, N. et al. (2009) The carboxyl terminus of Brca2 links the disassembly of Rad51 complexes to mitotic entry. Curr Biol 19, 1075-1085

79 Kabeche, L. et al. (2018) A mitosis-specific and R loop-driven ATR pathway promotes faithful chromosome segregation. Science 359, 108-114

80 ZIRKLE, R.E. and BLOOM, W. (1953) Irradiation of parts of individual cells. Science $117,487-493$

81 Mari, P.-O. et al. (2006) Dynamic assembly of end-joining complexes requires interaction between Ku70/80 and XRCC4. Proc Natl Acad Sci USA 103, 18597-18602

82 Britton, S. et al. (2013) A new method for high-resolution imaging of $\mathrm{Ku}$ foci to decipher mechanisms of DNA double-strand break repair. The Journal of Cell Biology 202, 579-595

83 Terasawa, M. et al. (2014) Canonical Non-Homologous End Joining in Mitosis Induces Genome Instability and Is Suppressed by M-phase-Specific Phosphorylation of XRCC4. PLoS Genet 10, e1004563

84 Royou, A. et al. (2010) BubR1- and Polo-coated DNA tethers facilitate poleward segregation of acentric chromatids. Cell 140, 235-245

85 Derive, N. et al. (2015) Bub3-BubR1-dependent sequestration of Cdc20Fizzy at DNA breaks facilitates the correct segregation of broken chromosomes. The Journal of Cell Biology 211, 517-532

86 Mason, J.M. et al. (1984) mu-2: mutator gene in Drosophila that potentiates the induction of terminal deficiencies. Proc Natl Acad Sci USA 81, 6090-6094

87 Latre, L. et al. (2003) Shortened telomeres join to DNA breaks interfering with their correct repair. Exp Cell Res 287, 282-288

88 Bailey, S.M. et al. (2004) Dysfunctional mammalian telomeres join with DNA doublestrand breaks. DNA Repair (Amst) 3, 349-357

89 McClintock, B. (1941) The Stability of Broken Ends of Chromosomes in Zea Mays. Genetics 26, 234-282 
90 Stephens, P.J. et al. (2011) Massive Genomic Rearrangement Acquired in a Single Catastrophic Event during Cancer Development. Cell 144, 27-40

91 Ly, P. and Cleveland, D.W. (2017) Rebuilding Chromosomes After Catastrophe: Emerging Mechanisms of Chromothripsis. Trends Cell Biol 27, 917-930

92 Wilkins, A.C. et al. (2019) The immunological consequences of radiation-induced DNA damage. J. Pathol. 247, 606-614

93 Glück, S. et al. (2017) Innate immune sensing of cytosolic chromatin fragments through cGAS promotes senescence. Nat Cell Biol 19, 1061-1070

94 Yang, H. et al. (2017) cGAS is essential for cellular senescence. Proceedings of the National Academy of Sciences 114, E4612-E4620

95 Harding, S.M. et al. (2017) Mitotic progression following DNA damage enables pattern recognition within micronuclei. Nature 548, 466-470

96 Mackenzie, K.J. et al. (2017) cGAS surveillance of micronuclei links genome instability to innate immunity. Nature 548, 461-465

97 Hatch, E.M. et al. (2013) Catastrophic nuclear envelope collapse in cancer cell micronuclei. Cell 154, 47-60 


\section{Highlights}

- DSBs that occur during mitosis do not induce cell cycle arrest and must be stabilized until the next G1 phase in order to avoid mis-segregation of acentric fragments and chromosomal instability

- The chromatin response to DSBs is rewired during mitosis. Chromatin ubiquitylation is blocked until late in mitosis and 53BP1-Shieldin is not recruited. Instead, TOPBP1 is recruited via direct interaction with MDC1 and this mechanism is required to prevent chromosomal instability.

- DSB repair by HR is inactive during mitosis. Some form of end joining activity may still be operational in mitosis or if DNA breaks are tethered until cells pass on in G1 where the breaks can be repaired by NHEJ.

- Micronuclei resulting from a failed DSB response during mitosis can induce chromothripsis and trigger an inflammatory response through activation of the cGASSTING signalling cascade. 


\section{Outstanding questions}

- Can breaks acquired during mitosis be repaired by an end-joining mechanism or is DSB repair completely blocked in mitosis? Could tethering of chromosome fragments during mitosis be a compensatory mechanism to prevent mis-segregation of acentric fragments and micronuclei formation?

- Are the mitotic kinases BubR1, PLK1 and Aurora B also implicated in tethering of acentric chromosome fragments in mammalian cells, similar to their Drosophila orthologues? And are these proteins recruited to sites of DSBs by $\gamma \mathrm{H} 2 \mathrm{AX}-\mathrm{MDC} 1-$ TOPBP1 in mitosis?

- What is the molecular function of TOPBP1 during mitosis? TOPBP1 is a classical adaptor protein that mediates protein-protein interactions. What protein interactions are mediated by TOPBP1 in mitosis?

- How are DSBs dealt with in later stages of mitosis, especially past the metaphase anaphase transition and how are unrepaired lesions transmitted into G1? 
Figures 1-3

Figure 1

(A)

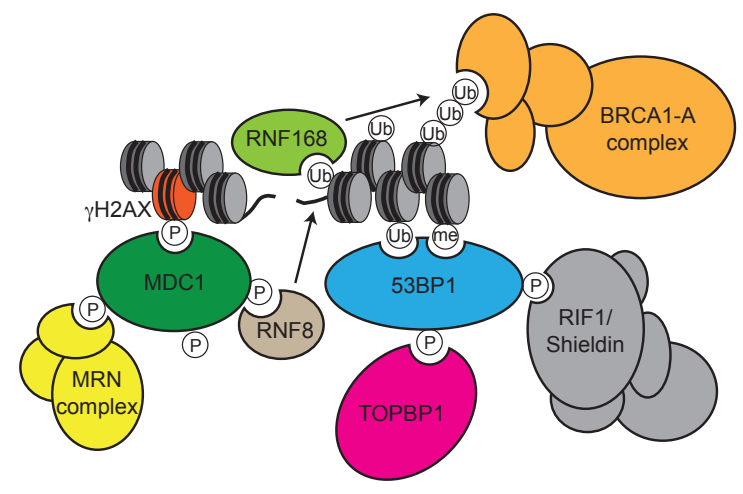

(B)

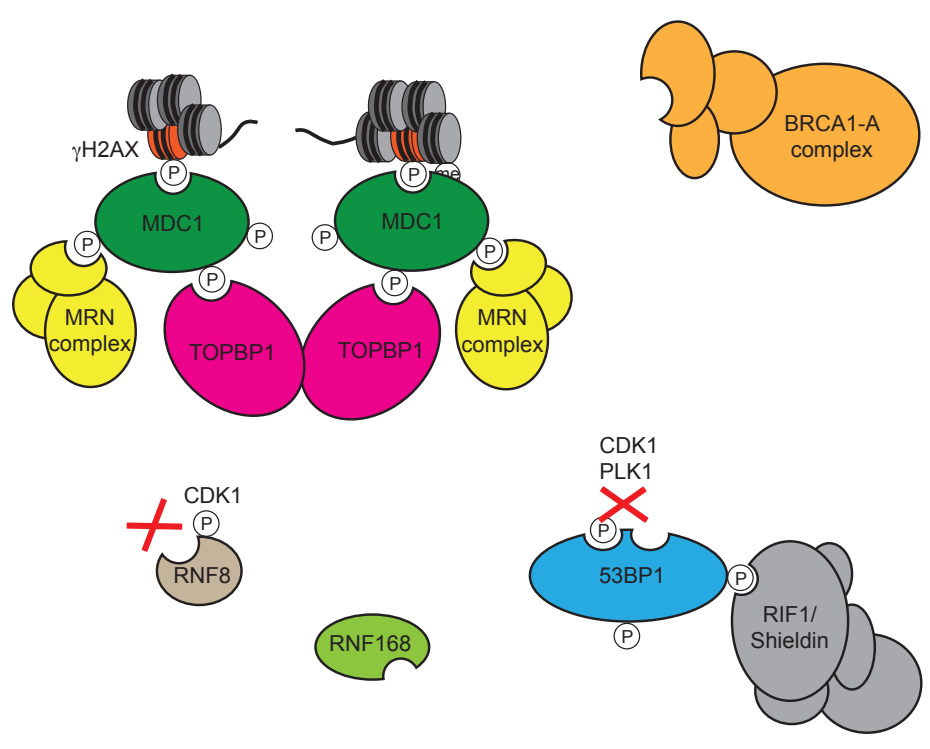


Figure 2

(A)
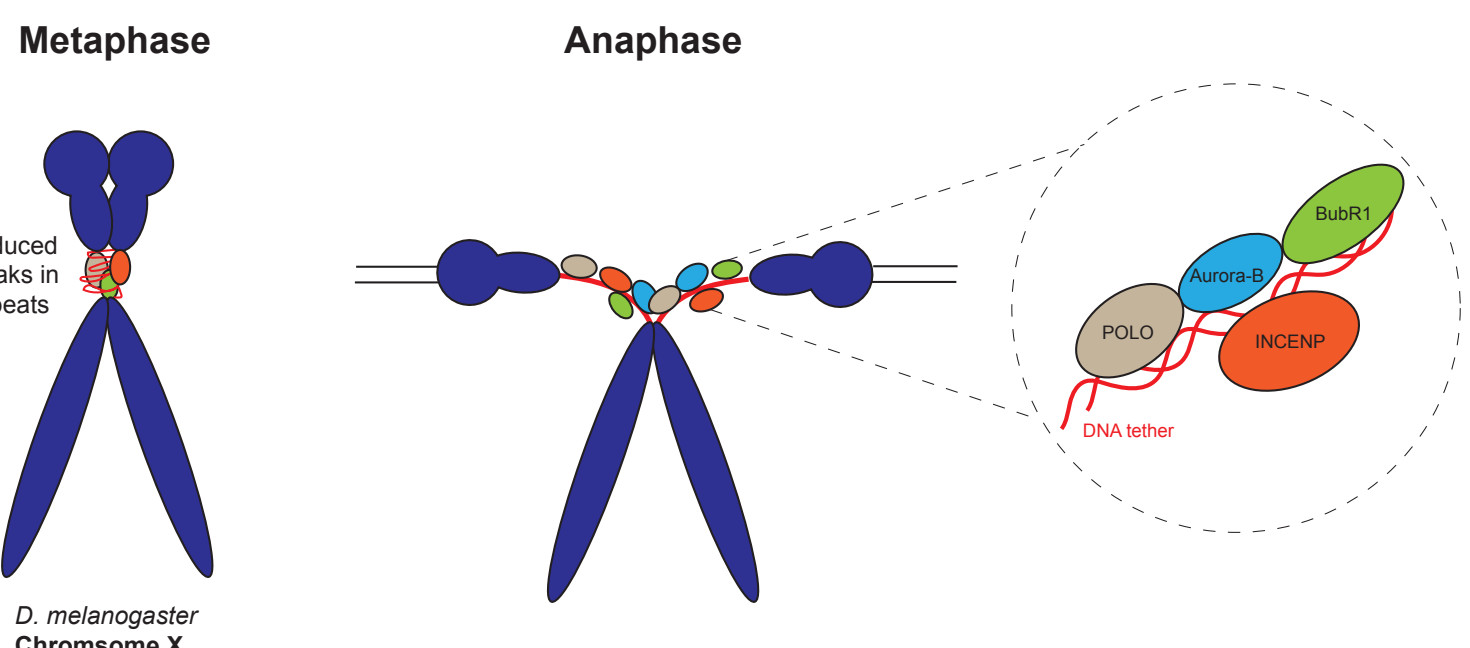

(B)

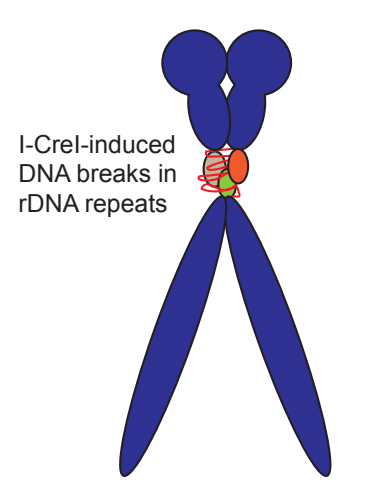

D. melanogaster Chromsome $\mathbf{X}$

Metaphase

Anaphase
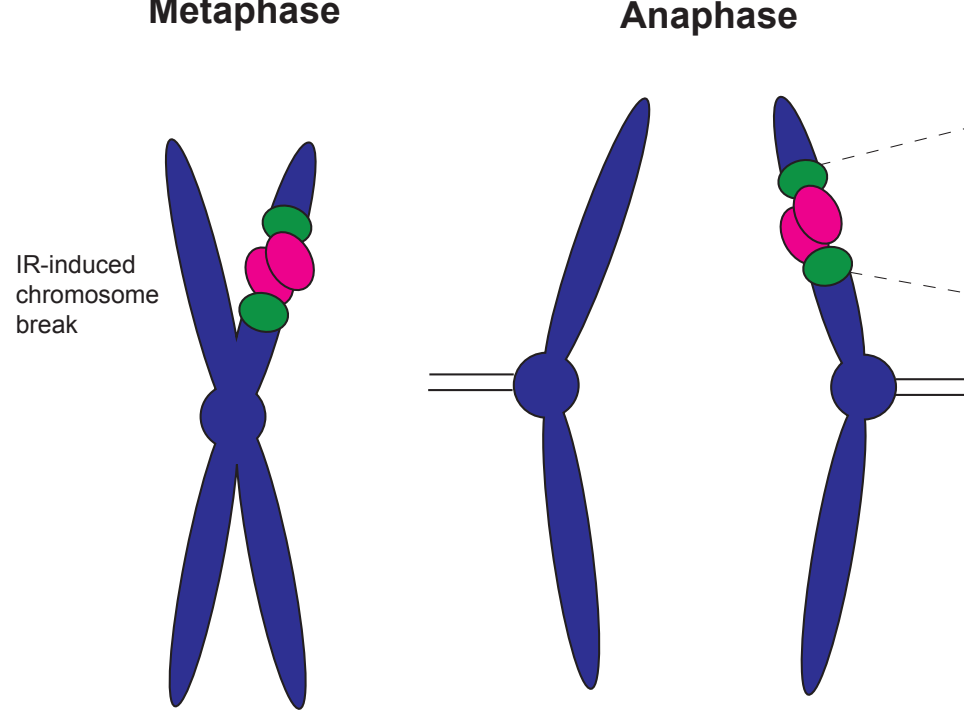


\section{Figure 3}
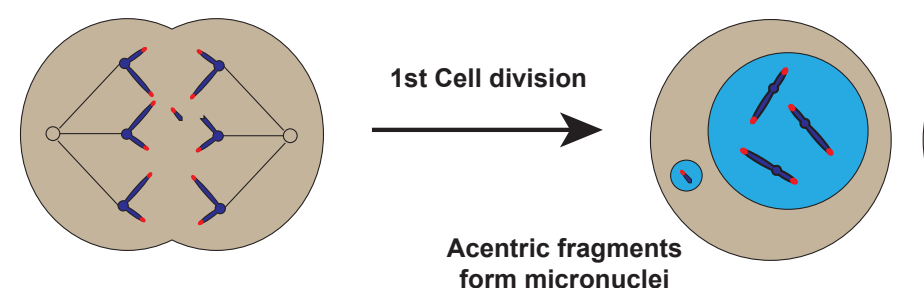

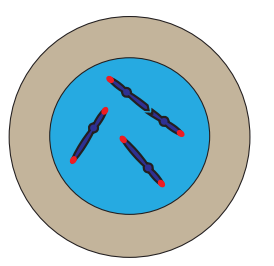

Centric fragments fuse and form dicentric chromosomes
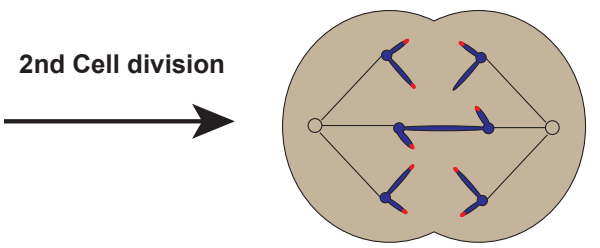

Anaphase chromosome bridges and BFB cycles

Chromothripsis

Re-incorporation

of micronuclei

in primary nucleus
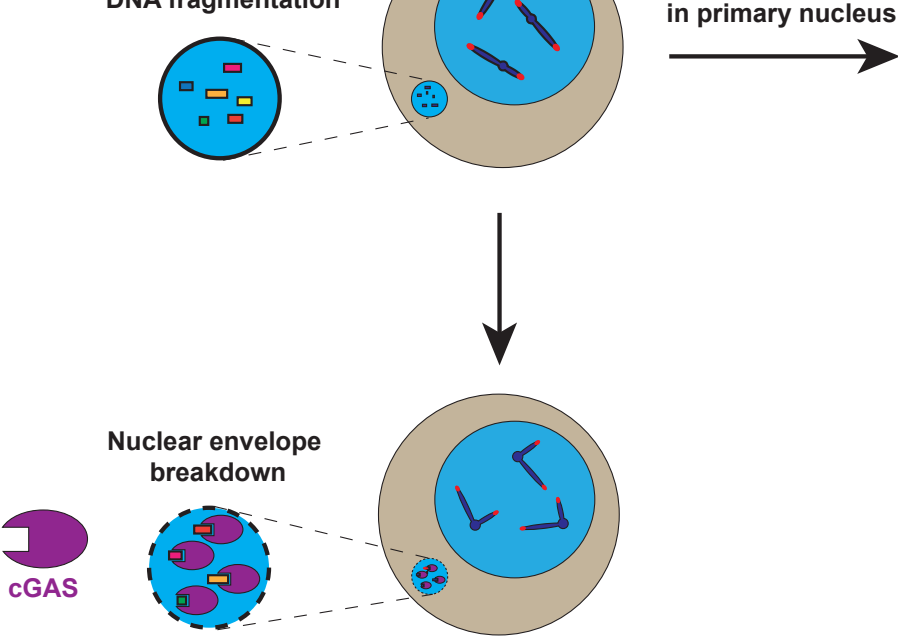

Activation of cGAS-STING-mediated inflammatory response 